\title{
Estudio de la climatología ecuatorial andina con métodos numéricos: pronósticos de tiempo, validaciones y reconstrucción de la atmósfera
}

\author{
Equatorial climatology study with numerical methods: \\ weather forecasting, validations and atmospheric reconstruction
}

\author{
Enrique Palacios*, Sheila Serrano*y Patricio Núñez
}

Centro de Investigación en Modelamiento Ambiental CIMA, Unversidad Politécnica Salesiana, Quito, Ecuador.

* Autores para correspondencia: wpalacios@ups.edu.ec y sserranov@ups.edu.ec.

Manuscrito recibido el 8 de noviembre de 2009. Aceptado, tras revisión, el 26 de noviembre de 2009.

\begin{abstract}
Resumen
La siguiente investigación, describe dos tipos de estudios acerca del clima y tiempo del Ecuador, implementando los modelos numéricos MM5 (Mesoescale Model versión 5) y WRF (Weather Research Forecasting Model), mostrando sus características principales e instalación para ajustarlo a las condiciones ecuatoriales. Los estudios que se realizan corresponden al pronóstico del tiempo del país, utilizando el modelo MM5 en variables como la temperatura, velocidad de viento y precipitaciones. El estudio de validación se lo hace sobre las precipitaciones de la ciudad de Quito a través de tablas de contingencia, estudiando los meses de agosto a octubre. Se logra así un POD (Probability of Detection) del $93,5 \%$ y un FAR (False Alarm Ratio) del I4\%, evidenciando la temporada inusualmente seca que se registró en esas fechas. Asimismo se presenta la primera parte del estudio de la reconstrucción de la atmósfera ecuatorial, utilizando el modelo WRF, mostrando el estudio de los perfiles verticales de las velocidades de viento; para concluir este estudio será necesario examinar las series temporales de al menos 30 años. Se obtienen resultados preliminares con una zona de vientos máximos a los 17 km de altura, visualizando sobre este nivel una disminución de su intensidad.
\end{abstract}

Palabras clave: modelos numéricos de mesoescala, climatología ecuatorial, predicción, validación,WRF.

\begin{abstract}
The present report describes two types of studies about the climate and weather in Ecuador by implementing the following numerical models: Mesoescale Mesoescale Model version 5 (MM5) and Weather Research Forecasting Model (WRF) showing its principal characteristics and installation by adjusting to equatorial conditions. These studies correspond to the weather forecasting in Ecuador, using MM5 model in variables like the temperature, wind speed and precipitations. The validation study is done on precipitations forecast of Quito by means of contingency tables, studying the months of august to october, thereby obtaining a POD (Probability of Detection) of $93.5 \%$ and a FAR (False Alarm Ratio) of I4\%, having demonstrated the unusually dry season that was registered at those dates. Also present the first part of the reconstruction of the equatorial atmosphere using WRF model, showing the study of the vertical profiles with wind speeds, in order to conclude this study will be necessary to examine the time series of at least 30 years. Preliminary results are obtained with a 17-kilometer zone of maximum winds, visualizing over this level a diminution of their intensity.
\end{abstract}

Keywords: mesoscale numeric models, equatorial climatology, forecasting, validation,WRF.

Forma sugerida de citar:

Palacios, E., S. Serrano y P. Núñez. 2009. Estudio de la climatología ecuatorial andina con métodos numéricos: pronósticos de tiempo, validaciones y reconstrucción de la atmósfera. La Granja.Vol. 10 (2). Pp. 16-26. ISSN: 1390-3799. 


\section{Introducción}

El conocimiento del clima y la predicción del tiempo han sido los motores fundamentales del desarrollo de la meteorología moderna. Desde la invención de los ordenadores en los años cuarenta, los modelos numéricos atmosféricos se han convertido en la herramienta fundamental del proceso de predicción del tiempo. Al mismo tiempo, los modelos numéricos se usan como herramienta de investigación de los procesos atmosféricos (García-Moya, 2004).

El objetivo más importante del estudio del clima es el de conocer con la máxima antelación y detalle posible el desencadenamiento de fenómenos adversos, es decir, de tipos de tiempo que puedan causar daños graves a la sociedad (grandes lluvias, sequías, temporales de viento, entre otros) sobre todo en estas épocas de cambios climáticos. Es importante señalar que actualmente no se concibe realizar predicciones del tiempo sin la ayuda de alguno de los múltiples modelos numéricos que existen en el mundo.

Un modelo atmosférico es una herramienta matemática construida sobre la base del conjunto de ecuaciones que gobiernan los movimientos en la atmósfera. Debido a que la atmósfera es un fluido pueden usarse las ecuaciones fundamentales de la dinámica de fluidos para resolver el problema de la evolución de los fenómenos meteorológicos en la atmósfera terrestre y dado que las citadas ecuaciones son diferenciales en derivadas parciales no lineales, no tienen solución exacta y deben resolverse por métodos numéricos. Así, desde un punto de vista puramente matemático, el problema de la predicción del tiempo es 'un problema de valores iniciales'. Por tanto, es necesario tener un conocimiento, lo más exacto posible, de la situación meteorológica en el instante inicial. Por esto, las comunicaciones han jugado un papel muy importante en el desarrollo de la predicción del tiempo. Mientras más desarrollados son los sistemas de comunicación, podemos disponer de las observaciones de todo el mundo y podemos realizar la predicción con el modelo numérico del que disponemos (García-Moya, 2004).

Además es necesario mencionar que un modelo atmosférico para la zona ecuatorial es un proyecto de investigación poco explorado, no sólo porque no ha sido de interés para países desarrollados, debido a su ubicación; sino porque la atmósfera ecuatorial es más gruesa y fenómenos, como la fuerza de Coriolis, se comportan de manera diferente.
Asimismo, a pesar de las herramientas numéricas y del asombroso poder de cálculo de los computadores, la naturaleza de la atmósfera se escapa de todos los métodos numéricos inventados debido a su comportamiento caótico -derivado de la no linealidad de las ecuaciones-. Conociendo sus condiciones iniciales se podría predecir el clima en el futuro, sin embargo, el caos de la atmósfera hace que una mínima diferencia en estas condiciones iniciales haga que el sistema evolucione de manera totalmente distinta. A este fenómeno se le conoce también como 'efecto mariposa', haciendo referencia al hecho de que el aleteo de las alas de una mariposa crea delicados cambios en las condiciones iniciales, los cuales durante el transcurso de un tiempo suficiente podrían modificarse hasta hacer que ocurra algo tan dramático como un tornado. Estos fenómenos ponen límite a la capacidad de realizar pronósticos a mediano y largo plazo que en su mayoría, después de los 3 días (en el mejor de los casos 7 días), adoptan un comportamiento inestable, que podría no reflejar la realidad de la atmósfera (Serrano et al., 2009).

\section{Materiales y métodos}

\section{I Descripción de MM5 y WRF}

Mesoescale Model versión 5, MM5 y Weather Research Forecasting Model,WRF son modelos que nacieron bajo las mismas premisas y grupos de investigación, es más, WRF es la versión avanzada de MM5 y básicamente todos los usuarios de MM5 ya han migrado a WRF, así que detallaremos el comportamiento de este último.

Se ha escogido WRF por ser un modelo numérico de mesoescala, cuyo código ha sido diseñado no sólo para representar los fenómenos físicos y dinámicos de la atmósfera, sino también para ser flexible, portable y eficiente en un entorno de cómputo paralelo y masivo. Se lo llama de mesoescala por el amplio espectro horizontal que ofrece, que va desde los metros hasta los miles de kilómetros. Sus aplicaciones incluyen la investigación, la predicción del tiempo numérica NWP (Numerical Weather Prediction), asimilación de datos, investigaciones en parametrizaciones físicas, simulaciones del clima en regiones pequeñas (downscaling), modelos de calidad de aire, modelamientos de acople océano-atmósfera y simulaciones ideales como por ejemplo remolinos en la capa límite, convección, ondas baroclínicas, entre otras.

Este modelo ha sido desarrollado por el trabajo cooperativo de muchas organizaciones de los Estados Unidos de América: el NCAR (National Center 
of Atmospheric Research), la división de Meterología de Mesoescala y Microescala (MMM), la NOAA (National Oceanic and Atmospheric Administration's), los centros nacionales para la predicción del tiempo NCEP (National Centers for Environmental Prediction), el Laboratorio para Sistemas de Predicción FSL (Forecast System Laboratory), el Departamento del Clima de la Fuerza Aérea AFWA (Air Force Weather Agency), el Laboratorio de Investigación Naval NRL (Naval Research Laboratory), el Centro de Análisis y Predicción de Tormentas de la Universidad de Oklahoma CAPS (Center for Analysis and Prediction of Storms) y la Administración de Aviación Federal FAA (Federal Aviation Administration), así como la participación de científicos de varias universidades.
Los principales componentes delWRF se describen en la Figura I, como se puede apreciar, este software provee una infraestructura tal que permite la resolución múltiple de ecuaciones dinámicas, así como la resolución de paquetes físicos que se conectan con estas ecuaciones a través de una interfaz física estándar; además posee programas de inicialización, y el sistema de asimilación de datos variacional WRFVar.Vale decir que dentro del sistema de resolución de las ecuaciones dinámicas, existen dos componentes: el WRF para Investigación Avanzada ARW (Advanced Research WRF) originalmente referido a la masa Euleriana, y el Modelo de Mesoescala No hidrostático NMM, el cual toma en cuenta movimientos verticales dentro de la atmósfera.

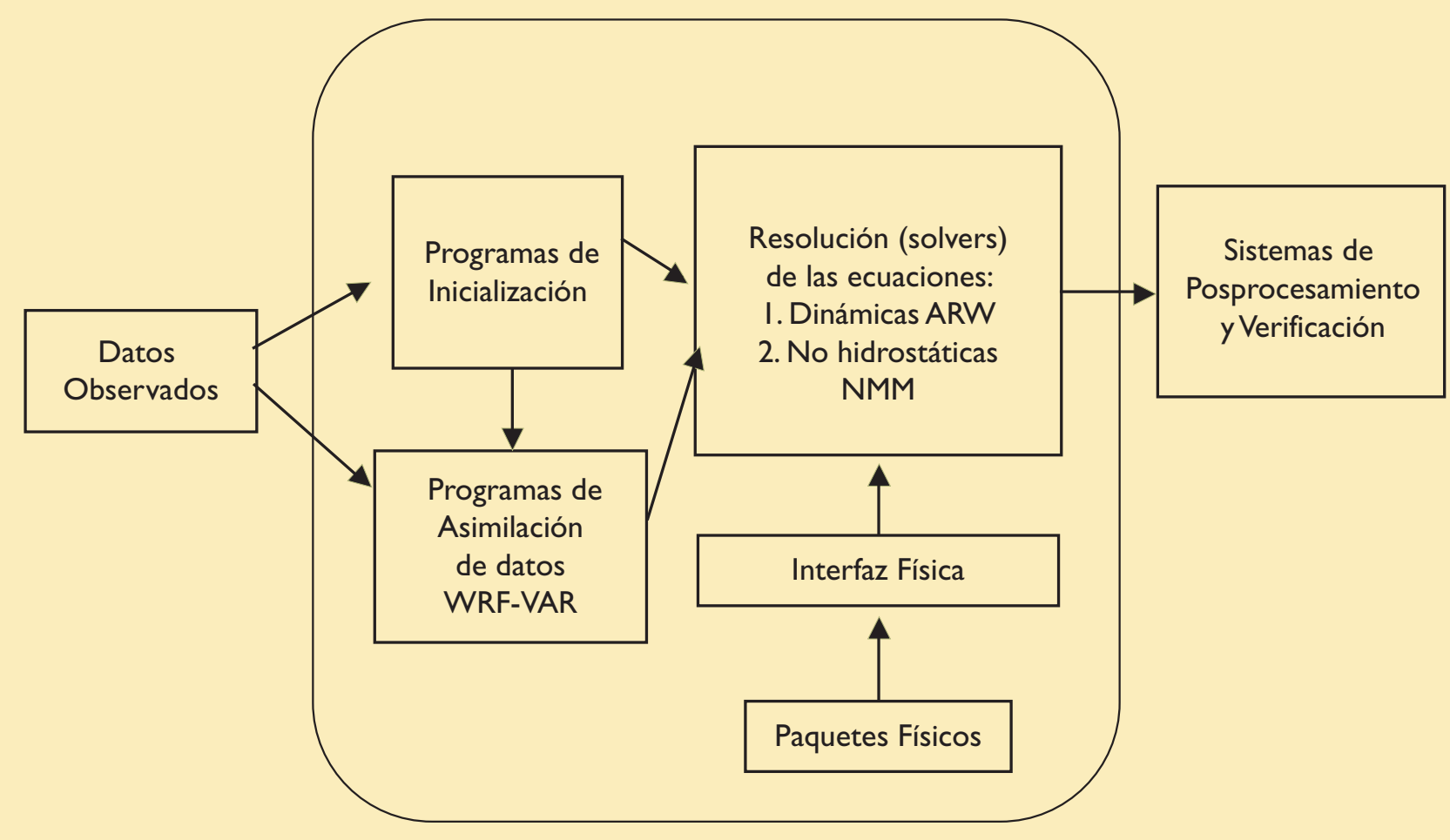

Figura I. Principales componentes y metodología del modelo numérico de mesoescala WRF

\section{I.I Ecuaciones que gobiernan el modelo}

El sistema que resuelve la parte dinámica ARW, resuelve ecuaciones de Euler no hidrostáticas y compresibles, se toman dentro de la dinámica de fluidos usando variables que tienen propiedades conservati- vas, (Ooyama, 1990). Estas ecuaciones son formuladas usando coordenadas verticales que tienen la propiedad de seguir la orografía del terreno (Laprise, 1992); y que además toman en cuenta la humedad, proyecciones sobre mapas curvos, Coriolis y otros términos de la curvatura (Haltiner y Williams, 1980). 


$$
\begin{array}{r}
\partial_{t} U+m_{x}\left[\partial_{x}(U u)+\partial_{y}(V u)\right]+\partial_{\eta}(\Omega u)+u_{d} \alpha \partial_{x} p+\left(\frac{\alpha}{\alpha_{d}}\right) \partial_{\eta} p \partial_{x} \phi=F_{U} \\
\partial_{t} V+m_{y}\left[\partial_{x}(U v)+\partial_{y}(V v)\right]+\left(\frac{m_{y}}{m_{x}}\right) \partial_{\eta}(\Omega v)+u_{d} \alpha \partial_{y} p+\left(\frac{\alpha}{\alpha_{d}}\right) \partial_{\eta} p \partial_{x} \phi=F_{V} \\
\partial_{t} W+\left(\frac{m_{x} m_{y}}{m_{y}}\right)\left[\partial_{x}(U w)+\partial_{y}(V w)\right]+\partial_{\eta}(\Omega w)-m_{y}^{-1} g\left[\left(\frac{\alpha}{\alpha_{d}}\right) \partial_{\eta} p-\mu_{d}\right]=F_{w} \\
\partial_{t} \theta+m_{y} m_{x}\left[\partial_{x}(U \theta)+\partial_{y}(V \theta)\right]+m_{y} \partial_{\eta}(\Omega \theta)=F_{\theta} \\
\partial_{t} \mu_{d}+m_{y} m_{x}\left[U_{x}+V_{y}\right]+m_{y} \partial_{\eta}(\Omega)=0 \\
\partial_{t} \phi+\mu_{d}^{-1}\left[m_{y} m_{x}[(U \partial)]_{x} \phi+V \partial_{y} \phi\right)+m_{y} \Omega \partial_{\eta} \phi-m_{y} g W(\Omega)=0 \\
\partial_{t} \mathrm{Q}_{m}+m_{y} m_{x} \partial_{x}\left(U q_{m}\right)+\partial_{y}\left(V q_{m}\right)+m_{y} \partial_{\eta}\left(\Omega_{q_{m}}\right)=\mathrm{FQ}_{m}
\end{array}
$$

Las ecuaciones presentadas son predictivas y conservativas, $F u, F v, F w$ y $F \theta$ las fuerzas en 3 dimensiones y rotacionales, respectivamente, derivadas de la física del modelo, mezclas turbulentas, proyecciones esféricas y la rotación de la tierra.

El parámetro $\eta$ es una coordenada vertical, también es llamada coordenada vertical de masa (mass vertical coordinate), es un parámetro que varía desde I en la superficie hasta 0 en el nivel más alto del dominio vertical del modelo. Al tomar en cuenta la humedad en la atmósfera se define de la siguiente manera:

$$
\eta=\left(p_{d h}-p_{d h t}\right) / \mu d
$$

Donde $p_{d h}$ y $p_{d h t}$ representan la presión hidrostática de la atmósfera seca y la presión hidrostática de la atmósfera seca en el borde superior de ésta. Mientras que $\mu_{d}(x, y)$ representa la masa de aire seco por unidad de área en $(x, y)$. Incluyendo este parámetro, se tiene que las variables de flujo apropiadas para las ecuaciones (I-7) son:

$U=\frac{\mu_{d} u}{m_{y}}, \quad V=\frac{\mu_{d} v}{m_{x}}, \quad W=\frac{\mu_{d} w}{m_{y}}, \quad \Omega=\frac{\mu_{d} \dot{\eta}}{m_{y}}, \quad \Theta=\mu_{d} \theta$
Donde $U, V$ y $W$ son las componentes del momentum utilizadas para obtener factores en la escala de mapa, en las dos direcciones horizontales y en la dirección vertical correspondientemente; mientras que, $w=\dot{\eta}$ es la velocidad vertical contravariante. Además, $\theta$ es la temperatura potencial, $\phi=g z$ es el geopotencial, $p$ es la presión, y $\quad \alpha_{d}=\frac{1}{\rho d}$ la densidad inversa del aire seco.

En el caso de los términos de curvatura, WRF permite 3 proyecciones sobre la esfera, la proyección conforme cónica de Lambert, que es una proyección cartográfica cónica, frecuentemente, usada en Navegación Aérea; la proyección estereográfica, muy usada para realizar mapas de las regiones polares de la Tierra; la proyección de Mercator, que es un tipo de proyección geográfica cilíndrica y proyecciones tipo latitud-longitud. Con este propósito, se requiere obtener proyecciones ortogonales sobre una malla (grid) en la Tierra, en donde $\Delta x$ y $\Delta y$ que son constantes puedan variar según la curvatura de la tierra, para esto se utiliza la transformación de la ecuación (I0):

$$
\left(m_{x}, m_{y}\right)=\frac{(\Delta x, \Delta y)}{\text { distancia sobre la tierra }}
$$

La descripción de las ecuaciones y el funcionamiento del modelo se encuentra detallado en la nota técnica del NCAR, escrita por Skamarock et al. (2008). 


\section{I.2 Parametrizaciones físicas}

Es importante mencionar que el modelo WRF incluye parametrizaciones físicas que abarcan casi en su totalidad las interacciones físicas que ocurren en la atmósfera, se las ha dividido en algunas categorías: microfísica, formación (parametrización) de cúmulus, física de la capa límite planetaria, física de la superficie del terreno, la radiación y difusión. A cada una de estas categorías se las ha incluido fuera de las ecuaciones dinámicas de Euler, ya que han sido desarrolladas por separado, y simplemente han sido agregadas como paquetes anexos a WRF. El desarrollo de estos paquetes no ha sido simple, la preparación de la física implicó llenar arreglos completos con las variables físicas requeridas que incluyen temperatura, presión, alturas, profundidades de capas y otras variables; todo tomando en cuenta los puntos de rejilla a mitad de nivel y en niveles completos. Estos paquetes físicos computan las tendencias para los componentes de la velocidad estandarizados, la temperatura potencial y los campos de la humedad.

En el caso de la microfísica, ésta incluye todo proceso relacionado con la precipitación: formación del vapor de agua y nube; mediante esquemas físicos simplificados adaptados a la física sofisticada de fases mezcladas; utilizados dentro del proceso de NWP. Dentro de estos esquemas o paquetes es necesario mencionar al de (Kessler, 1969) en el que se basó el modelo COMMAS de (Wicker y Wilhelmson, 1995), que estudia el vapor de agua, el agua en las nubes y la lluvia modelando los procesos de producción, caída y evaporación de la lluvia, así como el incremento y autoconversión del agua en la nube y su producción por condensación. Aunque en realidad la microfísica de los modelos atmosféricos ha sido desarrollada por muchos otros investigadores, de los cuales citaremos a los más importantes: Lin et al. (1983), Rutledge and Hobbs (1984), Tao et al. (1989) y Chen and Sun (2002), que trabajaron para dar paso a lo que se conoció como el esquema de Purdue Lin.Y otros como Hong et al. (2004) y Dudhia et al. (2008), que trabajaron en procesos dispersos pero muy valiosos.

Al referirnos a la formación de los cúmulus, se utilizan esquemas responsables de tomar en cuenta efectos sobre nubes convectivas y/o bajas a una escala menor a la de la rejilla tomada. Se estudia su formación mediante ajustes y esquemas de flujo de masa dentro del modelado del mesoescala. Estos esquemas representan flujos verticales debido al movimiento de corrientes ascendentes y descendentes, además del movimiento de compensación que se genera fuera de las nubes. Funcionan solamente en las columnas individuales que forman a las nubes, donde se adapta el esquema y se proporcionan perfiles de temperatura y humedad, además de tendencias en la formación de nubes y precipitaciones. Futuros esquemas pueden proporcionar las tendencias del comportamiento del momentum debido a su transporte convectivo. Todos estos aportes proporcionan el componente convectivo de la precipitación superficial. Se mencionan como principales contribuciones las generados dentro del esquema de Kain-Fritsch, (Kain, 2004) basado en Kain and Fritsch (1990) y Kain and Fritsch (1993). Así como el esquema Betts-Miller-Janjic (Janjic, 1994, 2000) y (Betts, 1986; Betts and Miller, 1986).

Las parametrizaciones correspondientes a la física de superficie calculan principalmente las velocidades de fricción y coeficientes de intercambio que permiten calcular los flujos de calor y humedad en la superficie; mediante modelos de superficie de tierra (land-surface models) y tensión superficial en la capa límite planetaria. Sobre superficies aquíferas estos flujos superficiales también se calculan. Estos esquemas no entregan tendencias, sino únicamente información que depende de la estabilidad del modelo. Este modelamiento se realiza a través de esquemas de superficie en múltiples capas, que incluyen estudios del comportamiento de la temperatura de la vegetación y humedad del suelo, además de tomar en cuenta formaciones aquiferas y zonas cubiertas de hielo tanto en tierra como en agua. Además es necesario mencionar que se incluye la física de la capa límite planetaria en donde se han tomado en cuenta esquemas no locales y predicciones del comportamiento energético en el caso kinético de las turbulencias. En este caso se han tomado en cuenta los trabajos desarrollados dentro de MM5 (Mesoescale Model V.5) por Paulson (1970), Dyer and Hicks (1970), y Webb (1970) quienes calcularon los coeficientes de intercambio en la superficie. Las velocidades convectivas fueron un aporte de Beljaars (1994) y Zhang \& Anthes (1982) y otros.

Cuando nos referimos a la radiación atmosférica, ésta es estudiada tomando en cuenta el amplio espectro de éstas, incluyendo tanto las bandas de ondas cortas así también como las largas; este esquema se adapta tanto a aplicaciones climáticas como de tiempo.Además se incluyen los efectos de las presencias de nubes y estelas, así como los flujos sobre la superficie del terreno. Esta parametrización incluye varios modelos que debemos mencionar: el Rapid Radiative Transfer Model (RRTM) Longwave, desarrollado por Mlawer et al. (1997), el cual representa el proceso de las ondas largas a través de va- 
por de agua, ozono, $\mathrm{CO}_{2}$,y trazas de otros gases, así como la profundidad de las nubes; el Eta Geophysica Fluid Dynamics Laboratory (GFDL) para ondas largas, desarrollado por Fels y Schwarzkopf (1975) y Schwarzkopf y Fels (1991) que incluyen cálculos sobre bandas espectrales; el modelo CAM, Community Atmosphere Model parametrización anterior desarrollada por el NCAR aplicado tanto para ondas cortas y largas, documentada plenamente por Collins et al (2004); el Eta Geopysical Fluid Dynamics Laboratory (GFDL) de onda corta, desarrollado principalemente por Lacis y Hansen (1974), el esquema incluido en MM5, desarrollado por Dudhia (1989) para onda corta, así como el de Goddard (Chou y Suarez, 1994) que también abarca el estudio de las ondas cortas en la atmósfera.

\subsection{Predicción del tiempo}

El estudio de la predicción del tiempo sobre el Ecuador, se realiza con el programa MM5 utilizando el sistema operativo Fedora, eligiendo 3 dominios anidados repartidos de la siguiente manera (Ver
Figura 2): el primer dominio, que comprende al Ecuador continental e Islas Galápagos, el segundo subdominio únicamente abarca al Ecuador continental, mientras que el tercer subdomino se refiere a la Sierra central.

La metodología en la obtención de los pronósticos, incluye la utilización de datos satelitales que se encuentran disponibles en el sitio web del NCAR, aunque encriptados, ya que sólo pueden ser descifrados por el sistema de preprocesamiento. Diariamente se requieren alrededor de $400 \mathrm{Mb}$, para inicializar los cálculos, lo cual indica la necesidad no sólo de una gran capacidad de cómputo, sino también de almacenamiento. Una vez logrados los datos, ya se cuenta con las condiciones iniciales necesarias para la resolución de las ecuaciones dinámicas y parametrizaciones físicas, tal como se indicó en el funcionamiento del modelo.

Las salidas del programa son visualizadas con el programa MM5toGRADS, pudiendo apreciar 16 parámetros meteorológicos: temperatura, rapidez y dirección del viento (en las tres direcciones), precipitación convectiva, precipitación no convectiva, humedad, hu-

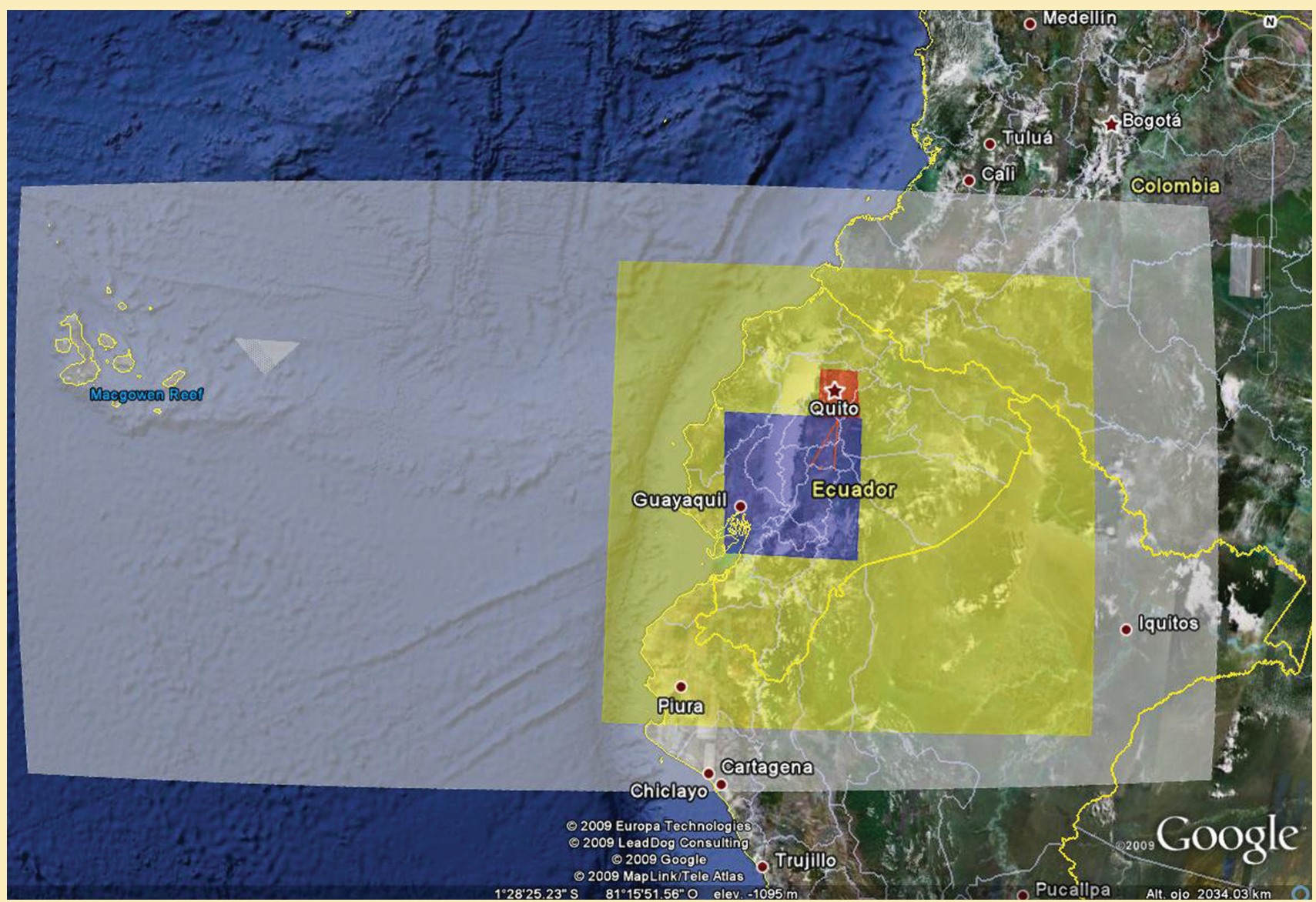

Figura 2. Dominios y subdominios ubicados sobre el Ecuador, aplicación sobre Google Earth. Fuente: Miguel Zambrano. 
medad relativa, evapotranspiración, uso de suelo, albedo, etcétera. Todas y cada una de estas variables pueden ser apreciadas a través de gráficos (Figuras 3, 4 y 5). Debido a que el interés se ha centrado en el comportamiento de la precipitación, la validación se ha realizado empezando por esta variable.

Los pronósticos tienen un alcance de 81 horas, de las cuales no se toman en cuenta las 9 primeras, debido a que el modelo aún no está estabilizado; ni las 9 últimas, que no reflejan confiablemente las variables de la atmósfera, debido al comportamiento caótico de ésta. Estos pronósticos son presentados como boletines informativos y almacenados para su futura validación y puede ser visualizados a través de la página web de la Universidad Politécnica Salesiana, www.ups.edu.ec.

\subsection{Validaciones}

El sistema de validación de los pronósticos se realiza a través de un sistema binario que designa por I y 0 a los eventos 'se registra precipitación' y 'no se registra precipitación' respectivamente; comparándolos con eventos 'se predice precipitación' y 'no se

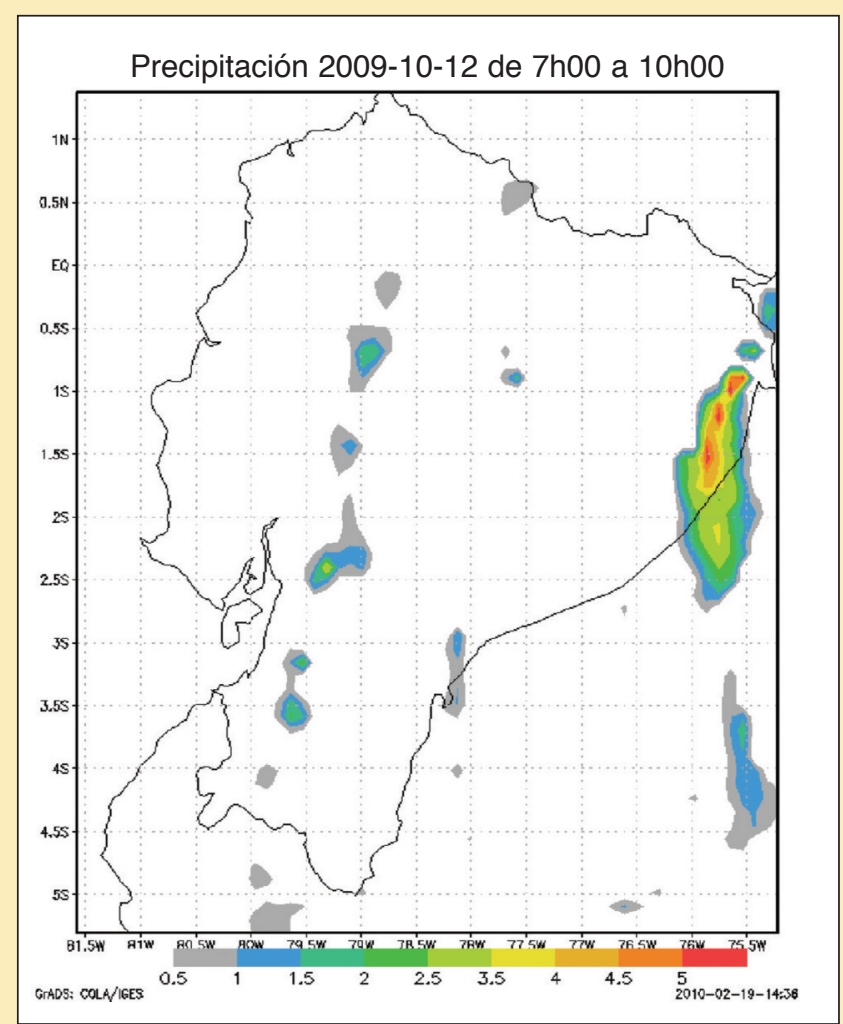

Figura 3. Pronóstico de precipitaciones correspondiente al día 12 de octubre de 2009 de 7 h00 a I0h00. En este caso, se prevén lluvias dispersas en el Ecuador continental, a excepción de la región oriental, con un máximo (en rojo) de 5 a $5,5 \mathrm{~mm}$ de lluvia.

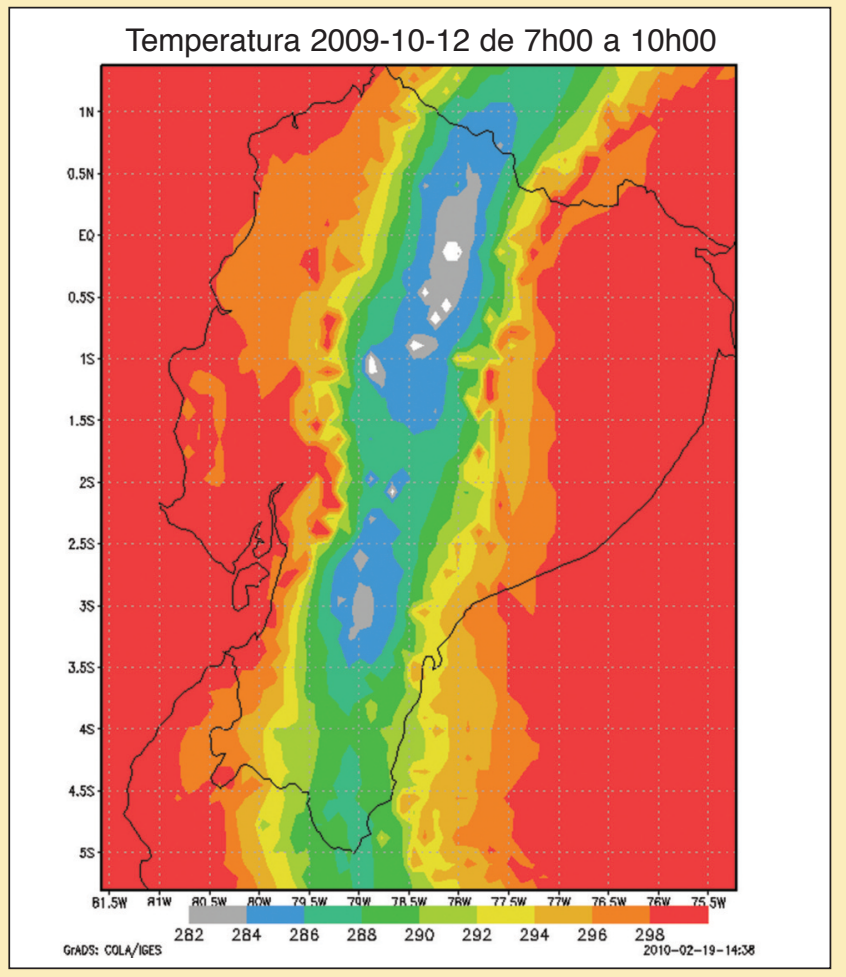

Figura 4. Pronóstico de temperaturas sobre el Ecuador continetal, del día 12 de octubre de 2009 de 7 h00 a 10 h00. $\mathrm{La}$ escala está en grados Kelvin $\left(1^{\circ} \mathrm{C}=1^{\circ} \mathrm{K}-273,15\right)$.

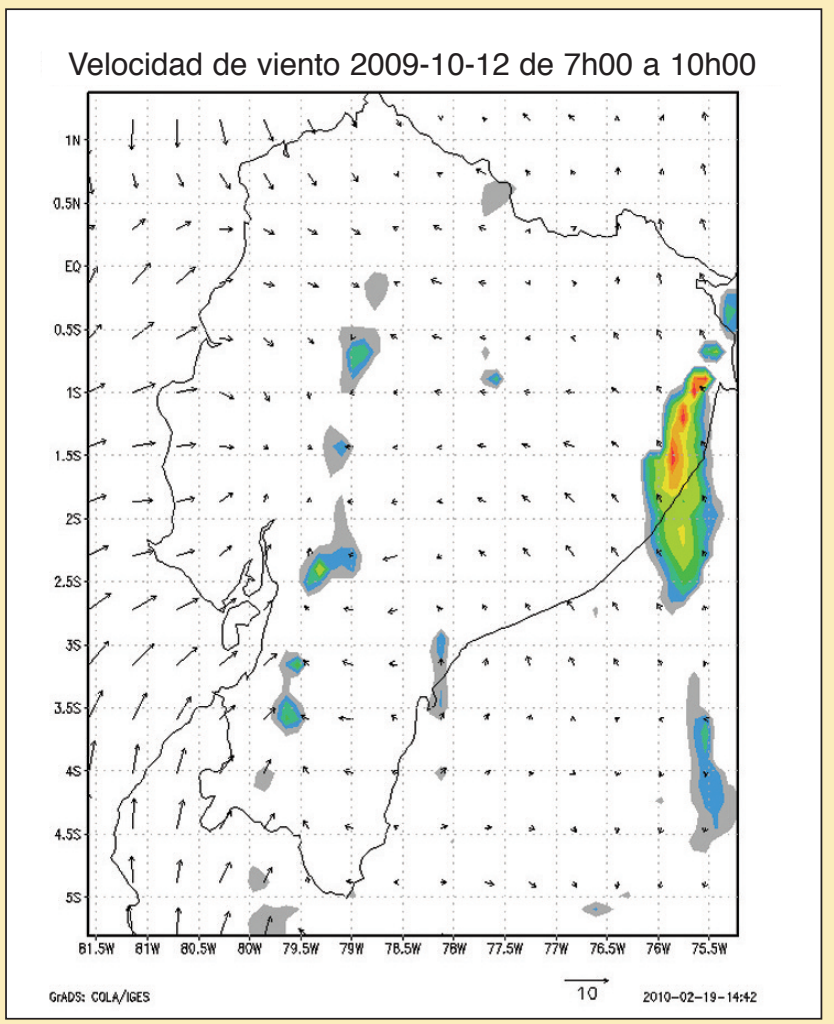

Figura 5. Velocidades del viento sobre el Ecuador continental del día 12 de octubre de 2009 de 7h00 a 10h00. Las flechas indican la dirección del viento, con una escala de $10 \mathrm{~m} / \mathrm{s}$ indicada en el gráfico. 
predice precipitación' que se designan asimismo con I y 0 , respectivamente. La precipitación fue observada directamente desde el campus universitario del Girón, en Quito, Universidad Politécnica Salesiana; correspondiente a los meses de agosto, septiembre y octubre de 2009.

Cada pronóstico abarca 3 horas, a éstos se los llama casos y se contabilizan en la tabla de contingencia (Ver Tabla I) el número de pronósticos acertados, no observados y nulos.

Tabla I.Tabla de contingencia con el número de pronósticos acertados, no observados, perdidos y nulos.

\begin{tabular}{lcc}
\hline & $\begin{array}{c}\text { Precipitaciones } \\
\text { Observadas } \\
(\mathrm{I})\end{array}$ & $\begin{array}{c}\text { Precipitaciones } \\
\text { No observadas } \\
(0)\end{array}$ \\
\hline $\begin{array}{l}\text { Precipitaciones } \\
\text { predichas (I) }\end{array}$ & 43 & 7 \\
$\begin{array}{l}\text { Precipitaciones } \\
\text { no predichas (0) }\end{array}$ & 3 & 597 \\
\hline
\end{tabular}

Como se puede observar, en estos meses se obtuvo un número exagerado (589) de pronósticos sin lluvia que sí fueron acertados, a éstos se los llama nulos ya que no son muy representativos para diagnosticar la capacidad predictiva de precipitación del modelo; es necesario indicar que el 2009 fue un año especialmente escaso de lluvias. Mientras, que los casos de lluvia y que sí fueron detectados (aciertos) fueron 43. Los casos en los que la precipitación se observa, pero que no se predice, sólo fueron 3; $y$, finalmente, los casos en los que se predice precipitación, pero ésta no se observa fueron 7 .

Los índices que definen la confiabilidad del modelo se calculan en referencia a los datos obtenidos de la tabla de contingencia, tomando como referencia (Tabla 2), los valores que definen las ecuaciones (II) a (I4).

Tabla 2. Valores asignados para el cálculo de los índices.

\begin{tabular}{lcc}
\hline & $\begin{array}{c}\text { Precipitaciones } \\
\text { Observadas }\end{array}$ & $\begin{array}{c}\text { Precipitaciones } \\
\text { No observadas }\end{array}$ \\
\hline $\begin{array}{l}\text { Precipitaciones } \\
\text { predichas }\end{array}$ & $\mathrm{a}$ & $\mathrm{b}$ \\
$\begin{array}{l}\text { Precipitaciones } \\
\text { no predichas }\end{array}$ & $\mathrm{c}$ & $\mathrm{d}$ \\
\hline
\end{tabular}

Con estos datos se definen los índices más importantes como la probabilidad de detección de precipitaciones (POD), por sus siglas en inglés, Probability of Detection, se define al dividir el número de veces que una precipitación fue predicha y observada sobre el número de veces que fue observada (Malvesada et al., 2006).

$$
P O D=\frac{a}{a+c}
$$

El índice de falsa alarma (FAR), por sus siglas en inglés, False Alarm Ratio, que se obtiene de dividir el número de veces que un suceso se predice y no se produce, sobre el número de veces que se predice (Malvesada et al., 2006).

$$
F A R=\frac{b}{a+b}
$$

Obviamente, el objetivo de todo modelo predictivo es maximizar el POD y disminuir el FAR. Existe además el índice de Heidke (HSS), por sus siglas en inglés, Heidke Skill Score, se trata de un índice que es relativamente alto cuando detecta eventos raros, se calcula de la siguiente forma:

$$
H S S=\frac{2(a d-b c)}{(d+c)(a+c)+(d+b)(a+b)}
$$

Un pronóstico perfecto tiene un HSS $=I$ y uno completamente aleatorio HSS $=0$ (Hennon et al., 2004). Sin embargo, uno de los índices más utilizados es el Índice de éxito crítico (CSI) Critical Success Index, por sus siglas en inglés, que nos da una medida más general de la capacidad de éxito del modelo numérico (Schaefer, 1990), se calcula dividiendo los pronósticos acertados para todas las precipitaciones predichas u observadas.

$$
C S I=\frac{a}{a+b+c}
$$

Con estos datos se generan los siguientes índices: 
Probabilidad de Detección (POD) 93,5\%, Radio de Falsa Alarma (FAR) I4,0\%, Índice de Heidke (HSS) $88,8 \%$ e Índice de éxito crítico (CSI) 81 , I\%

\subsection{Reconstrucción de la climatología de la tropósfera}

Es necesario aclarar la diferencia que existe entre tiempo y clima, el primero se refiere a una escala de tiempo muy corta que abarca días, y cuyo estudio ha sido históricamente responsabilidad de la meteorología que hace predicciones inmediatas y que, actualmente, como se ha dicho, se vale de métodos numéricos y computacionales. El clima, por otro lado se extiende a periodos de tiempo muy largos, ya que en estos lapsos es que tiende a ser regular, y determina de gran manera la evolución del ciclo geográfico de una región lo que permite el desarrollo de una determinada vegetación y un suelo perfectamente equilibrado (suelos climáticos). Pero, en periodos de tiempo geológicos, el clima también cambia de forma natural, los tipos de tiempo se modifican y se pasa de un clima a otro en la misma zona. Para definir un clima es necesaria la observación durante un lapso largo (la Organización Meteorológica Mundial estableció periodos mínimos de treinta años).

De esta manera, la determinación del clima del Ecuador es un arduo trabajo, que implica años de cálculos, en este análisis preliminar que comprende un año de trabajo, se tomarán en cuenta al menos 30 años de datos climatológicos ecuatorianos (Serrano et al., 2009b).

A futuro, la atmósfera en la zona de estudio reconstruida con WRF se deberá validar con datos obtenidos por las radiosondas.

\section{Conclusiones}

Se ha podido apreciar que el proceso de ajuste del modelo a la atmósfera ecuatoriana ha sido exitoso en lo que se refiere a la parte de predicción del tiempo ya que se han logrado Probabilidades de detección del $93,5 \%$, radios de falsa alarma bajos $14 \%$ y en general un índice de éxito crítico del $81,1 \%$. Cabe acotar que los resultados fueron favorables, aunque es necesario ampliar las validaciones en otros puntos del país y durante más tiempo para incrementar los índices de confianza.

\section{Agradecimientos}

Un sincero agradecimiento a las autoridades de la Universidad Politécnica Salesiana quienes hicieron posibles estas y otras investigaciones en Modelamiento numérico, a través de la II Convocatoria a Proyectos de Investigación. Asimismo un especial agradecimiento para Diego Villegas quien siempre estuvo dispuesto a darnos una mano con los problemas informáticos y a Miguel Zambrano por su colaboración con las imágenes.

\section{Referencias}

Beljaars, A.C.M., 1994. The parameterization of surface fluxes in large-scale models under free convection, Quart. J. Roy. Meteor. Soc., I21, 255-270.

Betts, A. K., 1986: A new convective adjustment scheme. Part I: Observational and theoretical basis. Quart. J. Roy. Meteor. Soc., II2, 677-691.

Betts, A. K., andM. J. Miller, 1986. A new convective adjustment scheme. Part II: Single column tests using GATE wave, BOMEX, and arctic air-mass data sets. Quart. J. Roy. Meteor. Soc. I I 2, 693-709.

Chen, S.-H., and W.-Y. Sun, 2002. A one-dimensional time dependent cloud model.J. Meteor.Soc. Japan, 80, 99-118.

Chou M.-D., and M. J. Suarez, 1994. An efficient thermal infrared radiation parameterization for use in general circulation models. NASA Tech. Memo. I04606, 3, 85pp.

Collins, W.D. et al., 2004. Description of the NCAR Community Atmosphere Model (CAM 3.0), NCAR Technical Note, NCAR/TN-464+STR, 226pp.

Dudhia, J., S.-Y. Hong, and K.-S. Lim, 2008: A new method for representing mixed-phase particle fall speeds in bulk microphysics parameterizations. J. Met. Soc. Japan, in press.

Dudhia, J., 1989. Numerical study of convection observed during the winter monsoon experiment using a mesoscale two-dimensional model, J.Atmos. Sci., 46, 3077-3I 07.

Dyer,A. J., and B. B. Hicks, 1970: Flux-gradient relationships in the constant flux layer, 
Quart.J. Roy. Meteor. Soc., 96, 7I5-72I.

Ecuador inmediato. 2008. SENACYT. Plataforma estratosférica de gran altitud pone a Ecuador en el límite de la ciencia. Disponible en línea: <http://www.ecuadorinmediato .com/Noticias/news_user_view/ecuadorinmediato_noticias-83052>

Fels, S. B. and M. D. Schwarzkopf, 1975. The Simplified Exchange Approximation: A New

Method for Radiative Transfer Calculations, J. Atmos. Sci., 32, |475-| 488.

García-Moya, J. 2004. Los modelos numéricos de predicción del tiempo. ACAM, Tethys. No. 2.

Haltiner, G. J., and R. T. Williams, 1980: Numerical prediction and dynamic meteorology. John Wiley \& Sons, Inc., 477pp.

Hennon, C., C. Marzban, J.S Hobgood. 2004. Improving Tropical Cyclogenesis Statistical Model Forecasts through the Application of a Neural Network Classifier. UCAR.

Hong, S.-Y., and Y. Noh, and J. Dudhia. 2006. A new vertical diffusion package with an explicit treatment of entrainment processes. Mon. Wea. Rev., I34, 23 I8-234I.

Hong, S.-Y., J. Dudhia, and S.-H. Chen, 2004: A Revised Approach to Ice Microphysical Processes for the Bulk Parameterization of Clouds and Precipitation, Mon. Wea. Rev., I32,

Janjic, Z. I., 1990: The step-mountain coordinate: physical package, Mon. Wea. Rev., II8, |429-| 443.

Janjic, Z. I., 1994: The step-mountain eta coordinate model: further developments of the convection, viscous sublayer and turbulence closure schemes, Mon. Wea. Rev., 122, 927-945.

Janjic, Z. I., 1996: The surface layer in the NCEP Eta Model, Eleventh Conference on Numerical Weather Prediction, Norfolk, VA, 19-23 August; Amer. Meteor. Soc., Boston, MA, 354-355.

Janjic, Z. I., 2000: Comments on "Development and Evaluation of a Convection Scheme for Use in Climate Models", J. Atmos. Sci., 57, p. 3686.

Janjic, Z. I., 2002: Nonsingular Implementation of the Mellor-

Kain, J. S., and J. M. Fritsch, 1990: A one-dimensional entraining/ detraining plume model and its application in convective parameterization, J. Atmos. Sci., 47, 2784-2802.
Kain, J. S., and J. M. Fritsch, 1993: Convective parameterization for mesoscale models: The Kain-Fritcsh scheme, The representation of cumulus convection in numerical models, K. A.

Kessler, E., 1969: On the distribution and continuity of water substance in atmospheric circulation, Meteor. Monogr., 32,Amer. Meteor. Soc., 84 pp.

Emanuel and D.J. Raymond, Eds., Amer. Meteor. Soc., 246 pp. Kain, J. S., 2004: The Kain-Fritsch convective parameterization: An update. J.Appl. Meteor., 43, I70-18I.

Lacis, A.A., and J. E. Hansen, 1974. A parameterization for the absorption of solar radiation in the earth's atmosphere. J.Atmos. Sci., 3I, II8-133.

José A. García-Moya Zapata, Los modelos numéricos de predicción del tiempo, Asociación Catalana de Meteorología, Thetys, No. 2, 2006.

Laprise R., 1992: The Euler Equations of motion with hydrostatic pressure as independent variable, Mon. Wea. Rev., 120, 197-207.

Lin, Y.-L., R. D. Farley, and H. D. Orville, 1983: Bulk parameterization of the snow field in a cloud model. J. Climate Appl. Meteor., 22, 1065-1092.

Malvesada, M.L, B. Gomez, E Penabad, G. Miguez, C. Balseiro,V. Perez-Muñuzuri. 2006. Resultados Preliminares de la Validación de WRF en Galicia. GFNL-USC. Meteogalicia.

Mlawer, E. J., S. J.Taubman, P. D. Brown, M. J. lacono, and S.A. Clough, 1997. Radiative transfer for inhomogeneous atmosphere: RRTM, a validated correlated-k model for the longwave.J. Geophys. Res., 102 (DI4), I6663-I6682.

Ooyama K. V., 1990: A thermodynamic foundation for modeling the moist atmosphere, J.Atmos. Sci., 47, 2580-2593.

Paulson, C.A., 1970: The mathematical representation of wind speed and temperature profiles in the unstable atmospheric surface layer. J. Appl. Meteor., 9, 857-86I.

Rutledge, S.A., and P.V. Hobbs, 1984: The mesoscale and microscale structure and organization of clouds and precipitation in midlatitude cyclones. XII: A diagnostic modeling study of precipitation development in narrow cloud-frontal rainbands. J.Atmos. Sci., 20, 2949-2972. 
Schwarzkopf, M. D., and S. B. Fels, I99I. The simplified exchange method revisited - An accurate, rapid method for computation of infrared cooling rates and fluxes. J. Geophys. Res., 96 (D5), 9075-9096.

Schaefer,J. 1990. The Critical Success Index as an Indicator of Warning Skill, AMS Journals On line.Vol.5(4). Pp. 570-575.

Serrano, S., E. Palacios, P. Núñez, J. Araujo. 2009. EI Modelamiento científico en la UPS: una alternativa en investigación, UNIVERSITAS, Año VI, No. 10

Serrano, S., E. Palacios, P. Núñez, M. Zambrano y C.Terán. 2009. Descripción de las mejores condiciones ambientales para el prototipo PGA: el modelo atmosférico. Boletín PGA. Skamarock,W., J. Klemp, J. Dudhia, D. Gill, D. Barker, M. Duda. X. Huang, W. Wang y J. Powers. 2008. A description of the advanced Research
WRF Version 3. NCAR Technical Note. NCAR/TN-475+SRT. Boulder. USA. I I 3pp.

Tao, W.-K., J. Simpson, and M. McCumber 1989: An ice-water saturation adjustment, Mon. Wea. Rev., I I7, 23।-235.

Webb, E. K., 1970: Profile relationships: The loglinear range, and extension to strong stability, Quart. J. Roy. Meteor. Soc., 96, 67-90.

Wicker, L. J., and R. B. Wilhelmson, 1995: Simulation and analysis of tornado development and decay within a three-dimensional supercell thunderstorm. J. Atmos. Sci., 52, 2675-2703.

Zhang, D.-L., and R.A. Anthes, 1982: A high-resolution model of the planetary boundary layer-sensitivity tests and comparisons with SESAME-79 data. J. Appl. Meteor., 2I, 1594-1609. 